\title{
Improving a diabetes foot service to prevent amputations: the Portsmouth experience
}

\author{
MALIK A HUMAYUN, DARRYL MEEKING
}

\begin{abstract}
Diabetes-related amputations have a dramatic effect on quality of life and life expectancy and are also a huge financial burden to the National Health Service (NHS). The city of Portsmouth had the second-highest amputation rate in the country from 2007-2010, according to data published in early 2011, leading to a restructuring of the foot care pathway in the area. Over a three year period, the rate of major amputations in people with diabetes in Portsmouth has fallen from $2.3 / 1,000$ to $1.4 / 1,000$, compared with the national average of $1.1 / 1,000$. Repeat hospital admissions for diabetic foot disease are comparable to the national average for the first time. We continue to identify areas of improvement needed for our patients with diabetes and hope to continue to improve foot outcomes for our patients.
\end{abstract}

Br J Diabetes Vasc Dis 2015;15:180-183

Key words: diabetic foot, ulceration, amputation

\section{Background}

Diabetes increases the risk of developing a wide range of foot complications, including foot ulceration. Major leg amputation is almost universally preceded by foot ulceration. Every $30 \mathrm{sec}-$ onds a lower leg is lost to diabetes somewhere in the world. ${ }^{1}$

Diabetes-related amputations have a dramatic effect on quality of life and life expectancy and are a huge financial burden to the NHS. The NHS spends $1636-639$ million per annum on diabetes-related ulceration and amputation, accounting for $0.6-0.7 \%$ of all NHS spending. ${ }^{2}$ The number of amputations in England has risen, from 5,700 in 2009/10 to more than 6,000 in 2010/11 and was expected to increase to more than 7,000 by 2014/15 if urgent action was not taken. ${ }^{3}$

The city of Portsmouth had the second-highest amputation rate in the country from 2007-2010, according to the data published in early 2011.4 The local diabetes service has identified

Department of Diabetes \& Endocrinology, Queen Alexandra Hospital Portsmouth, UK

Address for correspondence: Dr Malik A Humayun Specialist Registrar, Department of Diabetes \& Endocrinology, Queen Alexandra Hospital Portsmouth, Southwick Hill Rd, Portsmouth,

Hampshire PO6 3LY, UK

Tel: +44 (0)7588565966

E-mail: docmalik@me.com

http://dx.doi.org/10.15277/bjdvd.2015.038
Box 1. Local issues likely to have increased the risk of diabetes-related amputations

- There were inadequate multidisciplinary diabetic foot care clinics, therefore one clinic each week was provided in a location away from the major hospital site.

- A foot care pathway that directed patients with new diabetic foot ulcers and unexplained foot disease to a communitybased podiatry service and only on to a specialist multidisciplinary clinic when there was failure to heal or an added complication developed.

- A lack of dedicated educators on foot care for people with diabetes which contributed to a lack of self-care and awareness of foot problems.

- Low rates of referral and late referrals to specialist podiatrists. Our internal audit of diabetic patients undergoing major amputation revealed that only $50 \%$ of patients with major amputations had seen a podiatrist and fewer than 5\% had attended a multidisciplinary clinic.

- Low rates of foot examination in primary care and a lack of education for healthcare professionals on the identification and management of diabetic foot disease.

- Delayed and inadequate access to orthotic services for provision of appropriate insoles and/or footwear for pressure offloading.

- Poor access to orthopaedic specialists and limited access for "gold standard" offloading by "total contact casting" for patients with troublesome diabetic foot problems.

many of the problems leading to amputation and attempted to correct them, with the aim of reducing the amputation rates in our area. This has led to a restructuring of the foot care pathway, which we describe in this article.

Local issues that contributed to the high rate of diabetes-related amputations

A series of local problems were identified that were likely to have accounted for the high rate of diabetes-related amputations in Portsmouth (Box 1). These problems have been compounded by the fact that foot care is delivered by healthcare professionals working for three different healthcare providers in our locality.

\section{Service changes}

We commenced the "super six" model of diabetes care supported by a unique specialist-supported community model. ${ }^{5}$ The formation of the community diabetes team in 2010 led the way to improving diabetes management skills and education in primary care by supporting GP practices directly in their work place, 
as well as community nursing teams and patients within their own homes or local environment. The team provides direct dayto-day nursing support, twice-yearly community visits and telephone and email contact directly to nurse specialists and consultants, with the ownership of the patient remaining within the primary care setting.

We have pioneered four half-day education modules on foot disease each year since 2011 as part of a comprehensive diabetes education portfolio. These incorporate an explanation of the foot care pathway as it applies to a range of at-risk and diseased feet, a practical assessment of the foot, plus education on the management of diabetic foot disease and neuropathic pain. The courses are provided at no cost to all healthcare practitioners in our locality. They are currently supported by the pharmaceutical industry, although the education programme also receives financial support from Portsmouth Hospitals NHS Trust. We are exploring the option of these courses being commissioned and are planning to incorporate attendance towards the development of a Diabetes Practice Passport that endorses practices that deliver excellent diabetes care.

In 2011, we developed a diabetes care document for hospital inpatients to incorporate foot examination and a risk assessment for all patients with diabetes. Inpatient foot care was highlighted as a problem in the National Diabetes Audit and the use of this form is to be audited shortly.

The development of a new pathway of care (Box 2) was established to direct every patient with a diabetic foot ulcer or suspected acute Charcot disease to a specialist medical clinic comprising an advanced diabetes podiatrist and a diabetologist. The charts designed to guide foot management for people with diabetes are available online. ${ }^{6}$

There has also been a service commissioned to increase the frequency of physician-led clinics from one to three each week. Also, relocation of clinics to the acute hospital site has enabled rapid and easy access to the range of specialists required to deliver high-quality foot care. These changes have been accompanied by the introduction of a community diabetes team to support the delivery of routine diabetes care. This new model was introduced in Portsmouth in 2012 and provides healthcare

Box 2. Features of the restructured pathway of foot care in Portsmouth

- Within-24 hour radiology reporting

- Immediate microbiology advice (infection management)

- Immediate or early access to vascular surgery (consultant opinion and next-day rapid-access vascular clinics)

- Access to orthopaedic opinion and contact casting through the creation of additional urgent diabetes-related orthopaedic slots

- Improved access to orthotics services through fax and email referral

- Improved links to community podiatry with access to specific diabetes foot clinics and intensive therapy clinics

- Dedicated access to specialist nurse review for glycaemic control issues practitioners working with diabetes with:

- Immediate telephone advice from Monday to Friday from diabetes nurse specialists until 17.00 and from hospital consultants from 17.30-19.00;

- Advice from both diabetes nurse specialists and hospital consultants available by email within $24 \mathrm{~h}$;

- Twice-yearly $2 \mathrm{~h}$ visits to every GP surgery from the community team (a diabetes specialist nurse and a consultant) to be used according to the needs of each practice. Typically these sessions are used to update on service developments, to provide diabetes education, to provide advice on the management of specific patients, and to review complex patients.

Orthotic provision has been a problematic area and we have struggled to get timely access to appropriate offloading, including both the acute elements of this service (e.g. within $24 \mathrm{~h}$ provision of air-cast walkers) and the chronic elements (e.g. the fashioning of customised footwear). A private provider was introduced while orthotic services were tendered, resulting in significant improvements in service delivery.

Self-care is enormously important in both the identification of foot problems and to facilitate early treatment. Foot education is provided directly to patients at Diabetes Education and Self Management for Ongoing and Newly Diagnosed (DESMOND) educational courses, which are available for all new patients with type 2 diabetes in our area. Foot care issues will also be highlighted at refresher courses being set up to educate patients with pre-existing diabetes who may not have attended DESMOND or may require updates. A highly motivated group of patients in Portsmouth have set up an annual patient conference, which also provides an opportunity for an update on foot care issues.

\section{Early results}

The rate of major amputations in Portsmouth has fallen from $2.3 / 1,000$ to $1.4 / 1,000$ adults with diabetes over a three year period, compared with the national average of 1.1/1,000 (Figure 1, Table 1).., 7 The frequency of repeat hospital admissions for diabetic foot disease is comparable to the national average for the first time. ${ }^{8}$

Figure 1. Number of major amputations performed during a recent three-year period

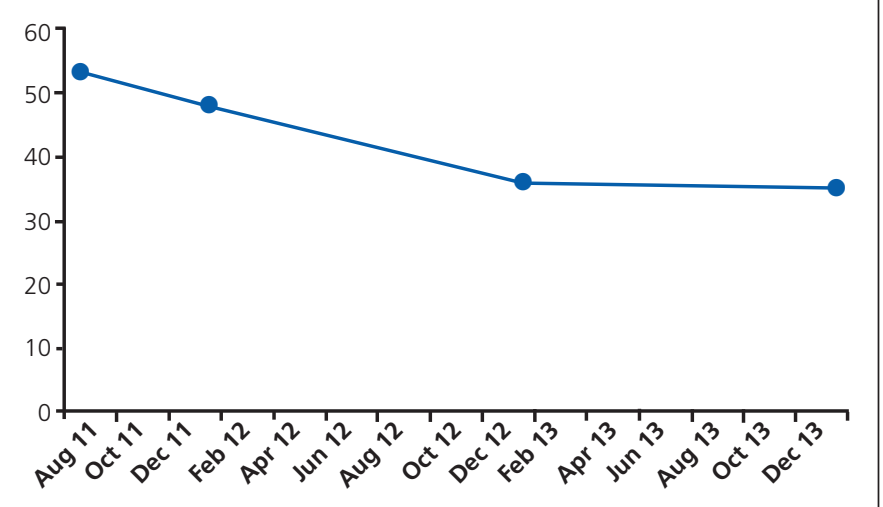


Table 1 Statistics for major amputations performed in Portsmouth City Teaching (PCT) area, compared with England as a whole, ${ }^{7}$ over a recent three-year period

\begin{tabular}{|c|c|c|c|c|c|c|c|c|c|c|}
\hline & \multicolumn{2}{|c|}{$\begin{array}{l}\text { Major amputations } \\
\text { performed during the } \\
\text { three year period per } \\
1,000 \text { adults with diabetes }\end{array}$} & \multicolumn{2}{|c|}{$\begin{array}{l}\text { Annual amputations } \\
\text { per } 1,000 \text { adults } \\
\text { with diabetes }\end{array}$} & \multicolumn{2}{|c|}{$\begin{array}{c}\text { Episodes of care } \\
\text { where an amputation } \\
\text { is performed on those } \\
\text { with diabetes }\end{array}$} & \multicolumn{2}{|c|}{$\begin{array}{l}\text { Episodes of care } \\
\text { for diabetic foot } \\
\text { diseases }\end{array}$} & \multicolumn{2}{|c|}{$\begin{array}{l}\text { Annual episodes of } \\
\text { care for diabetic } \\
\text { foot disease per } 1,000 \\
\text { adults with diabetes }\end{array}$} \\
\hline & РCT & England & РCT & England & РCT & England & РCT & England & РCT & England \\
\hline Aug 2011 & 2.3 & 1.08 & 5.7 & 2.7 & 133 & 180,80 & 629 & 116,884 & 27.1 & 17.6 \\
\hline Jan 2013 & 1.4 & 0.9 & 4.3 & 2.6 & 110 & 190,66 & 574 & 134,761 & 22.7 & 18.3 \\
\hline Jan 2014 & 1.3 & 0.9 & 4.3 & 2.6 & 113 & 200,30 & 574 & 143,503 & 21.6 & 18.6 \\
\hline
\end{tabular}

Box 3. Future vision

- We do not have an inpatient diabetes podiatry service which means that vascular surgeons provide debridement and surgical wound care. Consequently, our inpatient foot care pathway requires that all patients admitted with foot problems are admitted under surgery and all newly identified foot problems are reviewed within 24 hours by the surgical team. This may not be the best model of care for reducing the number of amputations.

- With the tendering of orthotic services it is essential that diabetes foot care is prioritised with early access to high quality offloading.

- There may need to be an increase in the number of physician-led clinics to meet capacity and provide a five, six or seven day physician-led foot service. National guidance is that acute diabetic foot problems should be seen by a diabetic foot multidisciplinary team within $24 \mathrm{~h}$. This may dovetail with our current work with Portsmouth Hospitals to move towards seven-day diabetes cover.

- We need to improve links with colleagues in vascular surgery to enable multidisciplinary discussion of patients being considered for major amputation.

- We need to further improve links with orthopaedic surgery to enable quick access to contact casting where appropriate and to facilitate better discussion of complex patients with diabetic foot problems.

- We need to look for innovative ways of engaging patients to improve self-care and the early identification and management of at-risk and ulcerated feet.

- The community podiatry team is working with commissioners to reduce the waiting time for podiatry review for at-risk and high-risk patients.

\section{Discussion}

The majority of diabetes-related amputations are preceded by foot ulceration and amputations may be prevented by the early discovery and appropriate intervention during the at-risk stage. Comprehensive education of all patients about diabetic foot problems to improve self-care is important. Regular appropriate assessment in primary care will identify a person's foot risk and

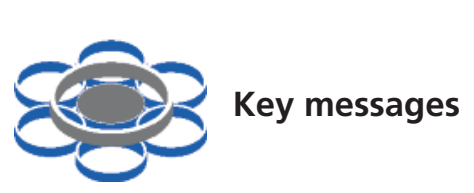

- Amputations may be prevented by the early detection and appropriate intervention during the at risk stage

- Once a foot ulcer has occurred early and appropriate intervention by a wide range of health care professionals (podiatrist, orthotist, tissue viability nurse, Diabetologist, vascular surgeon, orthopaedic surgeon, radiologist, microbiologist, community nurse and general practitioner) contribute to the reduction in amputation rates

- The Diabetic foot service is improving and a range of health care providers in Portsmouth are working jointly to provide appropriate resource and target it in a way that maximises improvement in foot outcomes for patients with diabetes

facilitate onward referral to a foot ulcer prevention team. Once a foot ulcer has occurred, early and appropriate intervention by a broad range of healthcare professionals (podiatrist, orthotist, tissue viability nurse, diabetologist, vascular surgeon, orthopaedic surgeon, radiologist, microbiologist, community nurse and general practitioner) contribute to the reduction in amputation rates.

Improvements in some of these areas may have contributed to this dramatic reduction in amputation, but much work remains to be done to provide an optimised service for our local diabetes population. Box 3 summarises areas in which we would like to see further improvements.

\section{Conclusions}

Our diabetic foot service is improving and a range of healthcare providers in Portsmouth are working together to provide appropriate resources that are targeted it in a way that maximises improvement in foot outcomes for patients with diabetes. Major 
amputation rates remain above, but close to, the national average. Almost all (about 95\%) of amputations are preceded by the development of diabetic foot ulceration, which itself may not develop in many patients until decades after diagnosis. Accordingly, it would not be reasonable to expect an immediate reduction of amputation rates to the national average. Other recognised risk factors for foot ulceration are prevalent in Portsmouth, including socioeconomic deprivation and Caucasian heritage. We continue to identify areas of improvement and, by addressing these, we can continue to improve foot outcomes for our patients with diabetes.

\section{Conflict of interest None \\ Funding None}

\section{References}

1. International Diabetes Federation/International Working Group on the Diabetic Foot. Diabetes and foot care. Time to Act. A joint publication of the International Diabetes Federation and the International Working Group on the Diabetic Foot. www.idf.org/webdata/docs/T2A_Introduc- tion.pdf (accessed May 2015).

2. Diabetes UK. Putting feet first: Fast track for a foot attack: Reducing amputations February 2013. http://www. diabetes.org.uk/upload/News/Factsheet Footcare for people with diabetes.pdf/ (accessed May 2015).

3. The Information Centre for Health and Social Care. Hospital Episode Statistics 2007/08-2010/11. http://www.hscic.gov.uk/searchcatalogue? $q=$ title \%3A\%22Hospital+Episode+Statistics\%2C+Admitted+patient+car e+-+England $\% 22 \&$ size $=10$

4. National Cardiovascular Intelligence Network. Diabetes footcare activity profile. August 2011. http://www. yhpho.org.uk/diabetesprofilesfoot/default.aspx (accessed May 2015).

5. Goulder T, Kar P. Facillitating diabetes care - a community approach. BMJ Qual Improv Report 2013;2.

http://dx.doi.org/10.1136/bmjquality.u201112.w708

6. Portsmouth Diabetes. May 2015. Guidance charts for diabetic foot care: Diabetes Foot Referral Pathway, Diabetic Foot Management Guideline, Charcot Alert. Available at http://www.portsmouthdiabetes.co.uk/images/portsmouth/diabetesfootreferralpathwayjan2010webversion.pdf (accessed May 2015).

7. National Cardiovascular Intelligence Network (NCVIN). http://www.yhpho.org.uk/default.aspx?RID=182342 (accessed May 2015).

8. Diabetes footcare activity profile; March 2014. http://www.yhpho.org.uk/ diabetesprofilesfoot/default.aspx (accessed May 2015).

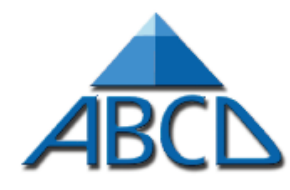

Association of British Clinical Diabetologists

\section{Canagliflozin (Invokana) Nationwide Audit Launching Soon!}

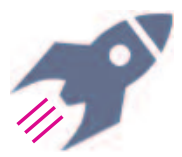

$A B C D$ is about to launch a nationwide audit of canagliflozin in the UK

to assess real clinical efficacy and safety \& inform future practice and guidelines

\section{Does your centre use canagliflozin (Invokana)?}

If yes, REGISTER YOUR CENTRE!

at http://www.diabetologists-abcd.org.uk/n3/Canagliflozin_Audit.htm

- you are invited to enter your patients' data into the bespoke online tool

- you will be able to analyse your local data easily

- the data will be automatically added to the national data in anonymised form

- we can provide easy-to-complete paper proformas for use in clinic if preferred

Please remember:

- the more data, the more complete our understanding of of canagliflozin in real clinical practice

- all contributors will be listed in publications arising from data submission 